\title{
Perfect independent sets with respect to infinitely many relations
}

\author{
Martin Doležal $^{1} \cdot$ Wiesław Kubiś ${ }^{1,2}$ (D)
}

Received: 17 October 2015 / Accepted: 12 July 2016 / Published online: 25 July 2016 (C) The Author(s) 2016. This article is published with open access at Springerlink.com

\begin{abstract}
We prove a result on perfect cliques with respect to countably many $G_{\delta}$ relations on a complete metric space. As an application, we show that a Polish group contains a free subgroup generated by a perfect set as long as it contains any uncountable free subgroup. This answers a recent question of Głąb and Strobin.
\end{abstract}

Keywords Perfect clique $\cdot$ Free subgroup · Open relation

Mathematics Subject Classification Primary 03E05 - 03E15; Secondary 54H05 · $20 \mathrm{E} 05 \cdot 20 \mathrm{~F} 38 \cdot 20 \mathrm{~K} 20$

\section{Contents}

1 Introduction . . . . . . . . . . . . . . . . . . . . . . . . . 848

2 Proof of the main result f . . . . . . . . . . . . . . . . . . . . . . 850

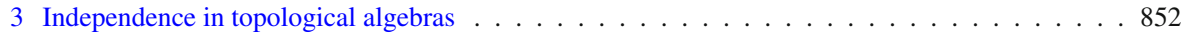

4 Other examples from group theory . . . . . . . . . . . . . . . . . . . 853

4.1 Free abelian subgroups . . . . . . . . . . . . . . . . . . . . . . 853

4.2 Torsion-free subgroups . . . . . . . . . . . . . . . . . . . . . . . . 854

4.3 Subgroups where all elements have a fixed order . . . . . . . . . . . . . . . . . . . . . . . . . . . . . . . . . . . . . . . . . . .

4.4 CA-subgroups . . . . . . . . . . . . . . . . . . . . . . 855

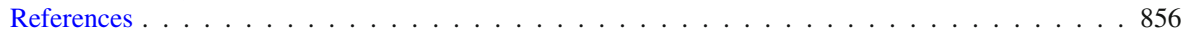

Research of Martin Doležal was supported by RVO: 67985840. Research of Wiesław Kubiś was supported by GAČR grant P201 14 07880S and RVO: 67985840.

$\triangle$ Wiesław Kubiś

kubisw@gmail.com

1 Institute of Mathematics, Czech Academy of Sciences, Prague, Czech Republic

2 Faculty of Natural Sciences, College of Science, Cardinal Stefan Wyszyński University in Warsaw, Warsaw, Poland 


\section{Introduction}

Given an $n$-ary relation $R$ on a set $X$, a subset $S$ of $X$ is said to be $R$-independent if for every sequence $s_{1}, \ldots, s_{n}$ of pairwise distinct elements of $S$ it holds that

$$
\left(s_{1}, \ldots, s_{n}\right) \notin R \text {. }
$$

This is one of the standard notions of independence considered widely in the literature, under various names. The above concept becomes more useful after generalizing to arbitrary families of relations. Namely, assuming $\mathcal{R}$ is a family of relations on $X$ (possibly, each of different arity), we say that $S \subseteq X$ is $\mathcal{R}$-independent if it is $R$-independent for every $R \in \mathcal{R}$. Independence with respect to a family of relations was considered (with a slightly more technical definition) by Mycielski [7].

Our aim is to present a dichotomy concerning the existence of independent sets in completely metrizable topological spaces. Here is our main result:

Theorem 1.1 Let $\kappa$ be a cardinal number, let $X$ be a completely metrizable space of weight $\kappa$, and let $\mathcal{R}$ be a countable family of $F_{\sigma}$ relations on $X$. Then exactly one of the following two statements holds.

(S) There exists an ordinal $\gamma<\kappa^{+}$such that every $\mathcal{R}$-independent set has CantorBendixson rank $\leq \gamma$ (that is, $\gamma$ th Cantor-Bendixson derivative of every $\mathcal{R}$-independent set is empty).

(P) There exists a perfect $\mathcal{R}$-independent set.

In this paper, we call a set $P$ perfect if it is nonempty, completely metrizable and has no isolated points. It is more usual to call a nonempty set perfect if it is closed and has no isolated points. However, every nonempty completely metrizable set without any isolated points contains a topological copy of the Cantor set. Therefore $(\mathrm{P})$ can be equivalently reformulated as 'there exists a perfect (in the usual sense) $\mathcal{R}$-independent set', or even 'there exists a topological copy of the Cantor set which is $\mathcal{R}$-independent'.

Note that the existence of a nonempty (even countable) dense-in-itself $\mathcal{R}$ independent set already implies $(\mathrm{P})$. Furthermore, $(\mathrm{P})$ holds whenever there exists an $\mathcal{R}$-independent set of cardinality $>\kappa$, as such a set always contains a dense-in-itself subset of cardinality $>\kappa$.

Special case of Theorem 1.1 has already been proved by the second author [5], under the assumptions that $X$ is separable and $\mathcal{R}=\{R\}$, where $R \subseteq X^{n}$ is a symmetric relation.

Theorem 1.1 is parallel to the following result of Mycielski:

Theorem 1 ([7]) Let $X$ be a completely metrizable space without isolated points, and let $\mathcal{R}$ be a countable family of relations on $X$ such that each $R \in \mathcal{R}$ is of the first category. Then there exists a perfect $\mathcal{R}$-independent set.

Clearly, every first category relation is contained in a first category $F_{\sigma}$ relation. Thus, Mycielski's theorem says that if (S) holds in our dichotomy then at least one of the relations is not meager.

It is necessary to point out that the dichotomy totally fails when increasing the Borel complexity of the relations. Namely, there exists a $G_{\delta}$ binary relation $R$ on the Cantor 
set $2^{\omega}$ such that every maximal $R$-independent set has cardinality exactly $\aleph_{1}$ and no perfect set is $R$-independent. A concrete example of such a relation was found by Vejnar and the second author [6, Thm. 2.1], although its existence was proved earlier by Shelah [8].

On the other hand, concerning a single binary relation, there is a significantly stronger result proved by Feng [3] for separable spaces and by Chaber and Pol [2] for arbitrary spaces:

Theorem 2 Let $X$ be a continuous image of a complete metric space of weight $\kappa \geq \aleph_{0}$, and let $R \subseteq X^{2}$ be a closed symmetric relation containing the diagonal of $X$. Then either $X=\bigcup_{\alpha<\kappa} X_{\alpha}$ such that each $X_{\alpha}^{2} \subseteq R$ for every $\alpha<\kappa$ or there exists a perfect $R$-independent set.

In fact, Chaber and Pol formulated and proved a more technical statement implying the one above. Theorem 2 can be viewed as a far reaching generalization of the old and classical theorem of Suslin: every uncountable analytic set contains a perfect subset (just let $R$ be the diagonal of $X$ ).

A recent result of Głąb and Strobin [4] asserts that a countable product of countable groups either contains a free group of cardinality $2^{\aleph_{0}}$ or else all of its free subgroups are countable. They also ask whether such a dichotomy holds for all automorphism groups of countable first order structures. In both cases, the groups carry a natural Polish topology, therefore our dichotomy provides an affirmative answer to the question of Głąb and Strobin, providing additional information concerning the topological structure of sets of free generators. The details are explained in Sect. 3.

Clearly, our main result (Theorem 1.1) can be rephrased dually, in terms of $G_{\delta}$ relations. Namely, given a relation $R \subseteq X^{n}$, a set $S \subseteq X$ is an $R$-clique ${ }^{1}$ if $\left(s_{1}, \ldots, s_{n}\right) \in R$ whenever $s_{1}, \ldots, s_{n} \in S$ are pairwise distinct. Clearly, $S$ is an $R$-clique if and only if it is $R^{\prime}$-independent, where $R^{\prime}=X^{n} \backslash R$. The notion of an $\mathcal{R}$-clique, where $\mathcal{R}$ is a family of relations, is defined in the obvious way. Now our dichotomy can be reformulated as follows:

Theorem 1.2 Let $X$ be a completely metrizable space of weight $\kappa$, where $\kappa$ is an arbitrary cardinal, and let $\mathcal{R}$ be a countable family of $G_{\delta}$ relations on $X$. Then exactly one of the following two statements holds.

(S) There exists an ordinal $\gamma<\kappa^{+}$such that every $\mathcal{R}$-clique has Cantor-Bendixson rank $\leq \gamma$.

(P) There exists a perfect $\mathcal{R}$-clique.

The next section is devoted to the proof of this statement. Section 3 explains applications to general topological algebras (including free groups), while the last section contains specific examples from group theory.

\footnotetext{
${ }^{1}$ The name clique comes from graph theory, where $R$ is the edge relation.
} 


\section{Proof of the main result}

Recall that the weight of a topological space is the least cardinality of an open base of the space. A Polish space is a completely metrizable separable topological space. Recall that a Hausdorff topological space is analytic if it is a continuous image of a Polish space.

For any subset $A$ of a topological space $X$ and for any ordinal $\gamma$, we denote by $A^{(\gamma)}$ the $\gamma$-th Cantor-Bendixson derivative of $A$. We also define the Cantor-Bendixson rank of $A$ as the least ordinal $\gamma$ such that $A^{(\gamma)}=\emptyset$. If such $\gamma$ does not exist, then the Cantor-Bendixson rank of $A$ is $+\infty$ which is, by definition, above all ordinals.

Proof of Theorem 1.2 First of all, we may assume that $\kappa \geq \aleph_{0}$. Indeed, if $\kappa<\aleph_{0}$ then $X$ is a finite discrete space, and so the Cantor-Bendixson derivative of every nonempty subset of $X=1$. Therefore (S) holds.

Let $\mathcal{R}=\left\{R_{n}\right\}_{n \in \omega}$. Then each $R_{n}$ is the intersection of countably many open relations $R_{n}^{m}, m \in \omega$. If we put $\tilde{\mathcal{R}}=\left\{R_{n}^{m}\right\}_{n, m \in \omega}$ then $\mathcal{R}$-cliques and $\tilde{\mathcal{R}}$-cliques coincide. Therefore without loss of generality, we may suppose that $\mathcal{R}$ consists of open relations.

Suppose that $X$ contains $\mathcal{R}$-cliques of Cantor-Bendixson rank arbitrarily close to $\kappa^{+}$. Let $\mathcal{R}=\left\{R_{n}\right\}_{n \in \omega}$ and let $r_{n}$ be such that $R_{n} \subseteq X^{r_{n}}$. Fix a complete metric $\varrho \leq 1$ on $X$ and fix a base $\mathcal{B}$ of $X$ of cardinality $\leq \kappa$. We construct inductively a Cantor scheme $\left\{U_{s}\right\}_{s \in 2<\omega} \subseteq \mathcal{B}$ with the following properties:

(1) $\operatorname{diam}\left(U_{s}\right) \leq 2^{- \text {length }(s)}$ for $s \in 2^{<\omega}$, and $\overline{U_{s}} \subseteq U_{t}$ whenever $t \in 2^{<\omega}$ is an initial segment of $s \in 2^{<\omega}$.

(2) For every $k \in \omega$, for every $\gamma<\kappa^{+}$there exists an $\mathcal{R}$-clique $A_{k, \gamma}$ such that

$$
A_{k, \gamma}^{(\gamma)} \cap U_{s} \neq \varnothing
$$

whenever $s \in 2^{k}$.

(3) For every $k \in \omega$, for every $i \leq k$, for every pairwise distinct $s_{1}, \ldots, s_{r_{i}} \in 2^{k}$ it holds that

$$
U_{s_{1}} \times \cdots \times U_{s_{r_{i}}} \subseteq R_{i}
$$

Note that (3) is automatically fulfilled when $r_{i}>2^{k}$. We start with $U_{\emptyset}:=X$ if $r_{0}>1$ and with $U_{\emptyset}:=R_{0}$ if $r_{0}=1$. Then (3) holds. The existence of $\mathcal{R}$-cliques of Cantor-Bendixson rank arbitrarily close to $\kappa^{+}$guarantees (2). Condition (1) holds by the choice of $\varrho$. Fix $n>0$ and suppose $\left\{U_{s}\right\}_{s \in 2^{<n}}$ has already been constructed.

Claim 2.1 Let $F$ be a finite $\mathcal{R}$-clique and fix $n \in \omega$. Then there exists a family $\left\{V_{x}\right\}_{x \in F}$ of pairwise disjoint open sets such that $x \in V_{x}$ for every $x \in F$ and such that for every $i \leq n$ and for every $S \in[F]^{r_{i}}$ it holds that

$$
\prod_{x \in S} V_{x} \subseteq R_{i}
$$


Proof We may suppose that there is $i \leq n$ such that $r_{i} \leq|F|$, otherwise this is trivial. For each $i \leq n$ and for each $S \in[F]^{r_{i}}$ choose a disjoint family of open sets $\left\{W_{x, i}^{S}\right\}_{x \in S}$ such that $x \in W_{x, i}^{S}$ for every $x \in S$ and such that

$$
\prod_{x \in S} W_{x, i}^{S} \subseteq R_{i}
$$

Let

$$
V_{x}=\bigcap\left\{W_{x, i}^{S}: i \leq n, S \in[F]^{r_{i}} \text { is such that } x \in S\right\} .
$$

Then $\left\{V_{x}\right\}_{x \in F}$ is as required.

Now, given $\gamma<\kappa^{+}$, choose $F_{\gamma} \subseteq A_{n-1, \gamma+1}^{(\gamma)} \cap \bigcup_{s \in 2^{n-1}} U_{s}$ such that $\left|F_{\gamma} \cap U_{s}\right|=2$ for every $s \in 2^{n-1}$. In particular, $\left|F_{\gamma}\right|=2^{n}$. Using the claim above, we find a disjoint family of open sets $\left\{V_{\gamma, x}\right\}_{x \in F_{\gamma}}$ such that $x \in V_{\gamma, x}$ for every $x \in F_{\gamma}$ and such that

$$
\prod_{x \in S} V_{\gamma, x} \subseteq R_{i}
$$

whenever $i \leq n$ and $S \in[F]^{r_{i}}$. Without loss of generality, we may assume that the closure of each $V_{\gamma, x}$ is contained in the appropriate $U_{s}$ (where $s \in 2^{n-1}$ is such that $x \in U_{s}$ ) and that $V_{\gamma, x} \in \mathcal{B}$ for every $x \in F_{\gamma}$ and $\gamma<\kappa^{+}$. We may also assume that each $V_{\gamma, x}$ has diameter $\leq 2^{-n}$. Using the fact that $\mathcal{B}$ has cardinality $\leq \kappa$, there is a cofinal set $C \subseteq \kappa^{+}$such that the family $\left\{V_{\gamma, x}\right\}_{x \in F_{\gamma}}$ is equal to some $\left\{V_{s}\right\}_{s \in 2^{n}}$ for every $\gamma \in C$, and $V_{s} \subseteq U_{s \uparrow(n-1)}$ for every $s \in 2^{n}$. Define $U_{s}:=V_{s}$ for $s \in 2^{n}$. Then $\left\{U_{s}\right\}_{s \in 2 \leq n}$ clearly satisfies (1) and (3). Condition (2) is witnessed by $A_{n, \gamma}:=A_{n-1, \gamma+1}$ whenever $\gamma$ is from the cofinal set $C$.

Finally, the Cantor set resulting from the scheme $\left\{U_{s}\right\}_{s \in 2^{<\omega}}$ is an $\mathcal{R}$-clique, thanks to condition (3).

We also note the following consequence of the main result.

Theorem 2.2 Let $\kappa$ be a cardinal, let $X$ be a continuous image of a completely metrizable space of weight $\leq \kappa$, and let $\mathcal{R}$ be a countable family of $G_{\delta}$ relations on $X$. If there exists an $\mathcal{R}$-clique of cardinality $\kappa^{+}$then there also exists a perfect $\mathcal{R}$-clique.

Proof Fix a continuous surjection $f: \tilde{X} \rightarrow X$, where $\tilde{X}$ is a completely metrizable space of weight $\leq \kappa$. Given $R \in \mathcal{R}$ of arity $n$, define

$$
\begin{array}{r}
\tilde{R}=\left\{\left(x_{1}, \ldots, x_{n}\right) \in \tilde{X}^{n}: f\left(x_{1}\right), \ldots, f\left(x_{n}\right)\right. \text { are pairwise distinct } \\
\text { and } \left.\left(f\left(x_{1}\right), \ldots, f\left(x_{n}\right)\right) \in R\right\} .
\end{array}
$$

Note that if $\left(x_{1}, \ldots, x_{n}\right) \in \tilde{R}$ then in particular $f\left\{\left\{x_{1}, \ldots, x_{n}\right\}\right.$ is one-to-one. The family $\tilde{\mathcal{R}}=\{\tilde{R}\}_{R \in \mathcal{R}}$ is clearly a countable family of $G_{\delta}$ relations on $\tilde{X}$. Suppose that $A \subseteq X$ is an $\mathcal{R}$-clique of cardinality $\kappa^{+}$. Choose $\tilde{A} \subseteq f^{-1}(A)$ such that for every $a \in \bar{A}$, it 
contains precisely one element from the preimage $f^{-1}(a)$. Then $\tilde{A}$ is an $\tilde{\mathcal{R}}$-clique of cardinality $\kappa^{+}$. Knowing that $\tilde{X}$ has weight $\leq \kappa$, it is easy to see that for every $\gamma<\kappa^{+}$, we have $\left|\tilde{A} \backslash \tilde{A}^{(\gamma)}\right| \leq \kappa$, and so $\tilde{A}^{(\gamma)} \neq \emptyset$. It follows that the Cantor-Bendixson rank of $\tilde{A}$ is $\geq \kappa^{+}$. Finally, Theorem 1.2 provides the existence of a perfect $\tilde{\mathcal{R}}$-clique $P \subseteq \tilde{X}$. Assuming that $P$ is compact, $f[P]$ becomes a perfect $\mathcal{R}$-clique in $X$.

Corollary 2.3 Let $X$ be an analytic space and let $\mathcal{R}$ be a countable family of $G_{\delta}$ relations on $X$. If there exists an uncountable $\mathcal{R}$-clique then there also exists a perfect $\mathcal{R}$-clique.

\section{Independence in topological algebras}

Recall that an abstract algebra is a structure of the form $(X, \mathcal{F})$, where $\mathcal{F}$ is a family of operations on the set $X$, where an operation is simply a function $f: X^{n} \rightarrow X$ with $n \geq 1$ (the number $n$ is the arity of $f$ ). A topological algebra is an algebra $(X, \mathcal{F})$, where $X$ is a topological space and all operations in $\mathcal{F}$ are continuous.

In order to define independence in abstract algebras, we need to recall the notion of a term and equation. Namely, assuming $\mathcal{F}=\left\{f_{i}^{X}\right\}_{i \in I}$, the language of $(X, \mathcal{F})$ is the set $\mathcal{L}=\left\{f_{i}\right\}_{i \in I}$ of formal operation symbols such that $f_{i}$ has the same arity as $f_{i}^{X}$. A term is, roughly speaking, an arbitrary formal operation that can be written as composition of formal operations from $\mathcal{L}$ and replacements of variables by other terms. The precise definition is recursive, of course. For details we refer to any textbook in model theory or logic. Given two terms $t_{1}, t_{2}$ with variables $\left(x_{1}, \ldots, x_{m}\right)$, the expression

$$
t_{1}\left(x_{1}, \ldots, x_{m}\right)=t_{2}\left(x_{1}, \ldots, x_{m}\right)
$$

is called an equation.

Now let $\mathcal{S}$ be a set of equations in $\mathcal{L}$ and let $(X, \mathcal{F})$ be an algebra whose language is $\mathcal{L}$. We say that $S \subseteq X$ is $\mathcal{S}$-independent if for every equation $t_{1}\left(x_{1}, \ldots, x_{m}\right)=$ $t_{2}\left(x_{1}, \ldots, x_{m}\right)$ in $\mathcal{S}$, it holds that

$$
t_{1}\left(s_{1}, \ldots, s_{m}\right) \neq t_{2}\left(s_{1}, \ldots, s_{m}\right)
$$

whenever $s_{1}, \ldots, s_{m} \in S$ are pairwise distinct. As an example, note that in the language of groups (where the operations are $\left(x_{1}, x_{2}\right) \mapsto x_{1} \cdot x_{2}, x \mapsto x^{-1}$, and the constant $x \mapsto$ 1) typical independence is with respect to all equations of the form $w\left(x_{1}, \ldots, x_{m}\right)=$ 1 , where $w$ ranges over all nontrivial reduced words (which are of course terms). Independent sets with respect to all these equations generate free subgroups.

Finally, notice that if $(X, \mathcal{F})$ is a topological algebra whose underlying topology is Hausdorff, then for every equation $t_{1}\left(x_{1}, \ldots, x_{m}\right)=t_{2}\left(x_{1}, \ldots, x_{m}\right)$ the relation

$$
R_{t_{1}, t_{2}}=\left\{\left(a_{1}, \ldots, a_{m}\right) \in X^{m}: t_{1}\left(a_{1}, \ldots, a_{m}\right)=t_{2}\left(a_{1}, \ldots, a_{m}\right)\right\}
$$

is closed. Clearly, $R_{t_{1}, t_{2}}$-independence is the same as independence with respect to the equation $t_{1}=t_{2}$. Evidently, if the language $\mathcal{L}$ is countable then the set of all equations is countable, too. Thus from our main result we obtain: 
Theorem 3.1 Let $(X, \mathcal{F})$ be a topological algebra of a countable language, whose underlying topology is completely metrizable and has weight $\kappa$, where $\kappa$ is an arbitrary cardinal. Let $\mathcal{S}$ be a fixed set of equations in the language of $(X, \mathcal{F})$. Then exactly one of the following possibilities hold.

(S) There exists an ordinal $\gamma<\kappa^{+}$such that $\gamma$ th Cantor-Bendixson derivative of every $\mathcal{S}$-independent subset of $X$ is empty.

(P) There exists a perfect $\mathcal{S}$-independent set.

In particular, $(\mathrm{P})$ holds whenever there exists a dense-in-itself $\mathcal{S}$-independent set.

As a concrete example, we turn to the variety of groups. The main consequences of Theorem 3.1 are the following.

Corollary 3.2 Let $G$ be a completely metrizable topological group. If $G$ has a densein-itself set of free generators then $G$ contains a free subgroup generated by a perfect set of free generators.

Corollary 3.3 Let $G$ be an analytic group. Then either all free subgroups of $G$ are countable or else $G$ contains a free subgroup generated by a perfect set of free generators.

Note that the above corollary can be applied to any group of the form $\operatorname{Aut}(M)$ with $M$ a countable first order structure, as such a group carries a natural Polish topology (namely, it is a closed subgroup of $S_{\infty}$, the countable infinite permutation group). This provides an affirmative answer to a question of Głąb and Strobin [4].

\section{Other examples from group theory}

In this section we demonstrate applications of our dichotomy to selected classes of groups.

\subsection{Free abelian subgroups}

It was proved in [1, Theorem 4] that every locally compact non-0-dimensional group contains a free abelian subgroup generated by a set of cardinality $\mathfrak{c}$. Here we provide examples of groups containing free abelian subgroups generated by a perfect set.

Theorem 4.1 Let $G$ be a completely metrizable topological group of weight $\leq \kappa$. If for every ordinal $\gamma<\kappa^{+}$there exists a set $S_{\gamma} \subseteq G$ of Cantor-Bendixson rank $\geq \gamma$ freely generating an abelian group, then $G$ has a free abelian subgroup generated by a perfect set of free generators.

Proof Let $R_{n}$ be the following $n$-ary relation: $\left(x_{1}, \ldots, x_{n}\right) \in R_{n}$ if and only if

$$
x_{1}^{k_{1}} \ldots x_{n}^{k_{n}} \neq 1
$$

whenever $k_{1}, \ldots, k_{n} \in \mathbb{Z} \backslash\{0\}$. Let $R$ be a binary relation defined by $\left(x_{1}, x_{2}\right) \in R$ if and only if $x_{1}, x_{2}, x_{1}^{-1}, x_{2}^{-1}$ commute with each other. Clearly, each $R_{n}$ is a $G_{\delta}$ 
relation and $R$ is a closed relation. It is also clear that a subset of $G$ freely generates a free abelian group if and only if it is an $\mathcal{R}$-clique, where $\mathcal{R}=\left\{R_{n}\right\}_{n \in \mathbb{N}} \cup\{R\}$. Thus, the statement above follows from Theorem 1.2.

Corollary 4.2 Let $G$ be a completely metrizable topological group. If $G$ has a free abelian subgroup which is generated by a dense-in-itself set of free generators then $G$ contains a free abelian subgroup generated by a perfect set of free generators.

Corollary 4.3 Let $G$ be an analytic group. Then either all free abelian subgroups of $G$ are countable or else $G$ contains a free abelian subgroup generated by a perfect set of free generators.

\subsection{Torsion-free subgroups}

Theorem 4.4 Let $G$ be a completely metrizable topological group of weight $\leq \kappa$. If for every ordinal $\gamma<\kappa^{+}$there exists a set $S_{\gamma} \subseteq G$ of Cantor-Bendixson rank $\geq \gamma$ generating a torsion-free (resp. abelian torsion-free) group, then $G$ has a perfectly generated torsion-free (resp. abelian torsion-free) subgroup.

Proof For $n, k \in \mathbb{N}$, let $R_{n, k}$ be the following $n$-ary relation: $\left(x_{1}, \ldots, x_{n}\right) \in R_{n, k}$ if and only if either

$$
w\left(x_{1}, \ldots, x_{n}\right)=1
$$

or

$$
\left(w\left(x_{1}, \ldots, x_{n}\right)\right)^{k} \neq 1
$$

whenever $w$ is any word with the domain $G^{n}$. We put $\mathcal{R}=\left\{R_{n, k}\right\}_{n, k \in \mathbb{N}}$. Clearly, each $R_{n, k}$ is a $G_{\delta}$ relation. It is also clear that a subset of $G$ generates a torsion-free group if and only if it is an $\mathcal{R}$-clique. Thus, the statement for general torsion-free subgroups follows from Theorem 1.2.

In the case of abelian torsion-free subgroups, we add one more binary relation $R$ defined by $\left(x_{1}, x_{2}\right) \in R$ if and only if $x_{1}, x_{2}, x_{1}^{-1}, x_{2}^{-1}$ commute with each other. This relation is clearly closed (and therefore $G_{\delta}$ ).

Corollary 4.5 Let $G$ be a completely metrizable topological group. If $G$ has a torsionfree (resp. abelian torsion-free) subgroup generated by a dense-in-itself set then $G$ contains a torsion-free (resp. abelian torsion-free) subgroup generated by a perfect set.

Corollary 4.6 Let $G$ be an analytic group. Then either all torsion-free (resp. abelian torsion-free) subgroups of $G$ are countable or else $G$ contains a torsion-free (resp. abelian torsion-free) subgroup generated by a perfect set. 


\subsection{Subgroups where all elements have a fixed order}

Theorem 4.7 Let $F \subseteq\{2,3, \ldots\}$ be a finite set. Let $G$ be a completely metrizable topological group of weight $\leq \kappa$. Iffor every ordinal $\gamma<\kappa^{+}$there exists a set $S_{\gamma} \subseteq G$ of Cantor-Bendixson rank $\geq \gamma$ generating a group where the order of each nonidentity element is in $F$, then $G$ has a perfectly generated subgroup where the order of each nonidentity element is in $F$.

Proof For $n \in \mathbb{N}$, let $R_{n}$ be the following $n$-ary relation: $\left(x_{1}, \ldots, x_{n}\right) \in R_{n}$ if and only for any word $w$ with the domain $G^{n}$ either $w\left(x_{1}, \ldots, x_{n}\right)=1$ or there is $k \in F$ such that

(i) $\left(w\left(x_{1}, \ldots, x_{n}\right)\right)^{i} \neq 1$ for $i=1, \ldots, k-1$,

(ii) $\left(w\left(x_{1}, \ldots, x_{n}\right)\right)^{k}=1$.

We put $\mathcal{R}=\left\{R_{n}\right\}_{n \in \mathbb{N}}$. Clearly, each $R_{n}$ is a $G_{\delta}$ relation. It is also clear that a subset of $G$ generates a group where the order of each nonidentity element is in $F$ if and only if it is an $\mathcal{R}$-clique. Thus, the statement follows from Theorem 1.2.

Corollary 4.8 Let $F \subseteq\{2,3, \ldots\}$ be a finite set. Let $G$ be a completely metrizable topological group. If $G$ has a dense-in-itself subgroup where the order of each nonidentity element is in $F$, then $G$ contains a perfectly generated subgroup where the order of each nonidentity element is in $F$.

Corollary 4.9 Let $F \subseteq\{2,3, \ldots\}$ be a finite set and let $G$ be an analytic group. Then either all subgroups of $G$ where the order of each nonidentity element is in $F$ are countable, or else $G$ contains a perfectly generated subgroup where the order of each nonidentity element is in $F$.

\subsection{CA-subgroups}

Recall that a group is said to be a CA-group (or a centralizer abelian group) if the centralizer of any nonidentity element is an abelian subgroup.

Theorem 4.10 Let $G$ be a completely metrizable topological group of weight $\leq \kappa$. If for every ordinal $\gamma<\kappa^{+}$there exists a set $S_{\gamma} \subseteq G$ of Cantor-Bendixson rank $\geq \gamma$ generating a CA-subgroup then $G$ has a perfectly generated CA-subgroup.

Proof For $n \in \mathbb{N}$, let $R_{n}$ be the following $n$-ary relation: $\left(x_{1}, \ldots, x_{n}\right) \in R_{n}$ if and only for any words $w_{i}, i=1,2,3$, where the domain of each $w_{i}$ is $G^{n}$ it holds under the notation

$$
\tilde{w}_{i}=w_{i}\left(x_{1}, \ldots, x_{n}\right), i=1,2,3,
$$

that

$$
\tilde{w}_{2}=1 \text { or } \tilde{w}_{1} \tilde{w}_{2} \neq \tilde{w}_{2} \tilde{w}_{1} \text { or } \tilde{w}_{3} \tilde{w}_{2} \neq \tilde{w}_{2} \tilde{w}_{3} \text { or } \tilde{w}_{1} \tilde{w}_{3}=\tilde{w}_{3} \tilde{w}_{1} \text {. }
$$

We put $\mathcal{R}=\left\{R_{n}\right\}_{n \in \mathbb{N}}$. Clearly, each $R_{n}$ is a $G_{\delta}$ relation. It is also clear that a subset of $G$ generates a CA-group if and only if it is an $\mathcal{R}$-clique. Thus, the statement follows directly from Theorem 1.2. 
Corollary 4.11 Let $G$ be a completely metrizable topological group. If $G$ has a densein-itself $C A$-subgroup then $G$ contains a perfectly generated $C A$-subgroup.

Corollary 4.12 Let $G$ be an analytic group. Then either all CA-subgroups of $G$ are countable, or else $G$ contains a perfectly generated CA-subgroup.

Open Access This article is distributed under the terms of the Creative Commons Attribution 4.0 International License (http://creativecommons.org/licenses/by/4.0/), which permits unrestricted use, distribution, and reproduction in any medium, provided you give appropriate credit to the original author(s) and the source, provide a link to the Creative Commons license, and indicate if changes were made.

\section{References}

1. Balcerzyk, S., Mycielski, J.: On the existence of free subgroups in topological groups. Fund. Math. 44, 303-308 (1957)

2. Chaber, J., Pol, R.: Remarks on closed relations and a theorem of Hurewicz. Topol. Proc. 22, 81-94 (1997)

3. Feng, Q.: Homogeneity for open partitions of pairs of reals. Trans. Am. Math. Soc. 339, 659-684 (1993)

4. Głab̧, S., Strobin, F.: Large free subgroups of automorphism groups of ultrahomogeneous spaces. Colloq. Math. 140, 279-295 (2015)

5. Kubiś, W.: Perfect cliques and $G_{\delta}$ colorings of Polish spaces. Proc. Am. Math. Soc. 131, 619-623 (2003)

6. Kubiś, W., Vejnar, B.: Covering an uncountable square by countably many continuous functions. Proc. Am. Math. Soc. 140, 4359-4368 (2012)

7. Mycielski, J.: Independent sets in topological algebras. Fund. Math. 55, 139-147 (1964)

8. Shelah, S.: Borel sets with large squares. Fund. Math. 159, 1-50 (1999) 